\title{
Developing a minimally-invasive anaesthesiological approach to non-intubated uniportal video-assisted thoracoscopic surgery in minor and major thoracic surgery
}

\author{
Henning Starke ${ }^{1}$, Norman Zinne ${ }^{2}$, Andreas Leffler ${ }^{1}$, Patrick Zardo ${ }^{2}$, Jan Karsten ${ }^{1}$ \\ ${ }^{1}$ Department of Anaesthesiology and Intensive Care Medicine, Hannover Medical School, Hannover, Germany; ${ }^{2}$ Department of Thoracic and \\ Cardiovascular Surgery, Hannover Medical School, Germany \\ Contributions: (I) Conception and design: H Starke, J Karsten; (II) Administrative support: H Starke, A Leffler, J Karsten; (III) Provision of study \\ materials or patients: H Starke, N Zinne, P Zardo, J Karsten; (IV) Collection and assembly of data: H Starke, J Karsten, N Zinne; (V) Data analysis \\ and interpretation: All authors; (VI) Manuscript writing: All authors; (VII) Final approval of manuscript: All authors. \\ Correspondence to: Dr. Jan Karsten. Department of Anaesthesiology and Intensive Care Medicine, Hannover Medical School, Carl-Neuberg-Strasse 1, \\ 30625 Hannover, Germany. Email: Karsten.Jan@mh-hannover.de.
}

Background: Non-intubated uniportal video-assisted thoracoscopic surgery (niVATS) is a novel approach to major and minor lung resection. It benefits from a holistic anesthesiological concept with adequate pain relief and sedation in a minimal-invasive setup allowing thoracic procedures under spontaneous breathing. At present no anesthesiological gold standard for niVATS exists. The primary aim of our retrospective observational study was to evaluate feasibility and safety of minimally invasive niVATS for both minor and major pulmonary resections at our institution.

Methods: All 88 consecutive patients scheduled for niVATS minor or major thoracic procedures were included into the study. Anaesthesia was performed according to a departmental niVATS algorithm including both regional anaesthesia and sedation. Patient characteristics and early outcome data including intraoperative and postoperative findings were compared between groups. Prediction scores for postoperative complications (LAS VEGAS, ARISCAT, ThRCRI) were calculated and compared.

Results: No early mortality and a low overall morbidity rate of $28.4 \%$ were encountered. Conversion to orotracheal intubation was required in $6.8 \%$ of all cases. Postoperative pulmonary complications occurred in $15.9 \%$ of total cases and were lower than predicted by both LAS VEGAS and ARISCAT respectively. Cardiac complications were found in $1.1 \%$ and lower than predicted by ThRCRI. A persistent air leak occurred in $11.4 \%$ of total cases and was significantly higher in major resection. Postoperative chest tube duration and hospital length of stay in the major resection group exceeded times reported by other groups.

Conclusions: niVATS appears to be safe in both minor and major thoracic procedures. A minimally invasive anaesthesiological approach foregoing central iv lines, arterial blood pressure measurement and urinary catheterization is feasible. Our niVATS protocol appears to be a viable alternative for both minor and major thoracic procedures in selected patients.

Keywords: Regional anaesthesia; intubation; thoracoscopy; postoperative pulmonary complications

Submitted Jun 03, 2020. Accepted for publication Oct 10, 2020.

doi: $10.21037 /$ jtd-20-2122

View this article at: http://dx.doi.org/10.21037/jtd-20-2122 


\section{Introduction}

Anecdotal evidence of non-intubated lung surgery was first reported in 1865 by Sir Francis Richard Cruise, who utilized a cystoscope developed by Maximilian Nietze for thoracoscopy in an empyema patient (1). After introduction of the double lumen tube by Eric Carlens in 1949, thoracic surgery was characterized by general anaesthesia and controlled mechanical ventilation (2). Thoracic procedures became increasingly less invasive with advent of modern video-assisted thoracic surgery (VATS) technology, and in 1992 Giancarlo Roviario reported the first minimally invasive anatomical lung resection (3). In 2004, nonintubated lung surgery was scientifically reintroduced and combined with minimally invasive techniques, resulting in Non-intubated uniportal video-assisted thoracoscopic surgery (niVATS) (4,5). Diego Gonzales-Rivas refined the technique to a single incision ("uniportal") approach, thus further reducing it's invasiveness, and uniportal niVATS was born (6-8).

Several studies and meta-analyses focussing on minimally invasive techniques showed benefits in short term outcomes like reduction of length of hospital stay (LOS), complication rates and even immunological response $(9,10)$. Recently, these findings were confirmed by a comprehensive metaanalysis by Yu et al. including 2,929 patients (11).

Despite an increasing interest in niVATS, no standard of anaesthesiological care for minor and major lung surgery has been proposed. Implemented protocols range from light sedation with local anaesthesia to general anaesthesia combined with epidural anaesthesia and maintained spontaneous ventilation with or without using a laryngeal mask. Procedures for local and regional analgesia include techniques like skin infiltration and single intercostal nerve blocks, compartment blockades like erector spinae plane block (ESPB) and serratus anterior blockade or neuroaxial blockades like paravertebral blockade (PVB) or thoracic epidural anaesthesia (TEA). Furthermore, most published studies report the use of invasive monitoring. Thus, an overall reduction of invasiveness is achieved by reducing the surgical trauma and maintaining spontaneous ventilation, without a truly minimally invasive anesthesiological approach.

Previous data collected from three major studies show a faster recovery and low complication rates for nonintubated procedures, while surgical results seem to be similar (12-14). But according to a European and a more recent German survey (German Thorax Registry), adoption of niVATS for major lung surgery in Europe still is very rare $(15,16)$. The biggest published case study from Hungary reports sixteen patients who underwent uniportal niVATS for anatomical lung resections (17).

We would like to report our initial experience with uniportal niVATS for minor and major lung surgery. We performed a retrospective analysis of our prospectively collected data to evaluate the effectiveness and safety of a standardized holistic anaesthesiological and surgical protocol for both minor and major lung surgery. We present the following article in accordance with the STROBE reporting checklist (available at http://dx.doi.org/10.21037/jtd-202122).

\section{Methods}

The study was conducted in accordance with the Declaration of Helsinki (as revised in 2013) and the Harmonized Tripartite Guideline for Good Clinical Practice from the International Conference on Harmonization. Our study protocol was approved by the local ethics committee (Hannover Medical School, Chair: Prof. S. Engeli, No. 8818_BO-K_2019) and need for individual patient consent was waived. This was a single-center retrospective cohort study of a prospectively maintained thoracic surgery database. 88 consecutive patients scheduled for niVATS from June 2018 until December 2019 were identified by chart review (Department of Anaesthesiology and Intensive Care Medicine, Department of Thoracic and Cardiovascular Surgery, Hannover Medical School, Germany). All patients $>18$ years of age scheduled for niVATS regardless of underlying disease were eligible for inclusion and divided into major $(n=40)$ or minor $(n=48)$ lung surgery groups. Major procedures included all forms of anatomical lung resection, ranging from segmentectomy to lobectomy, while minor procedures included wedge resection, pleurectomy or pleurodesis. Available patient characteristics and early outcome data including intraoperative and postoperative findings were collected. Exclusion criteria for niVATS, as proposed by our multidisciplinary working group, were age $<18$ years, ASA physical status $>$ IV, morbid obesity (body mass index $>35$ ), relevant airway secretions, persistent cough, hypoxemia $\left(\mathrm{PaO}_{2}<60 \mathrm{mmHg}\right)$, massive hypercapnia (with central hypoventilation syndrome or acute right heart failure), reflux, hemodynamic instability, need for lung separation (i.e.protection from contamination), difficult airway evaluated by the anesthesist, and neurological disorders (seizures, unable to cooperate, intracranial 


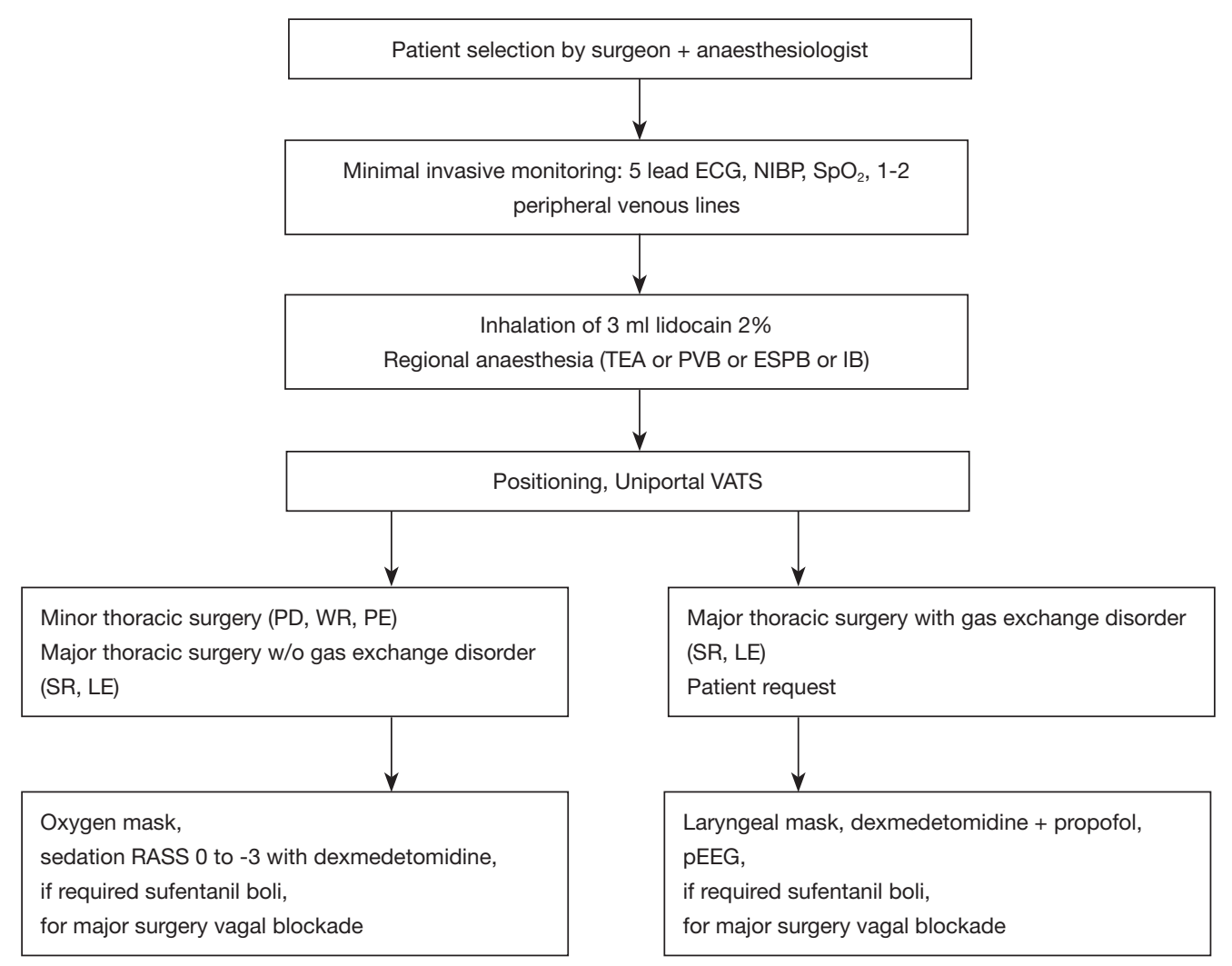

Figure 1 Flow diagram of perioperative care. ECG, electrocardiogram; NIBP, non-invasive blood pressure; SpO ${ }_{2}$, peripheral oxygen saturation; TEA, thoracic epidural anesthesia; PVB, paravertebral block; ESPB, erector spinae plane block; IB, intercostal block; PD, pleurodesis; WR, wedge resection; PE, pleural empyema; SR, segment resection; LE, lobectomy; RASS, Richmond Agitation Sedation scale; pEEG, processed electroencephalogram.

pressure $>15 \mathrm{mmHg}$ ).

\section{Anaesthesiological management}

Anaesthesia was performed according to our multidisciplinary niVATS algorithm (Figure 1). Standard monitoring includes ecg, non-invasive blood pressure measurement, pulse oximetry, respiratory rate, approximation of end-tidal carbon dioxide monitoring (etCO $\mathrm{C}_{2}$, i.e., modified respirography) and processed electroencephalogram (Narcotrend ${ }^{\mathrm{TM}}$, Hannover, Germany). All patients received 1-2 peripheral intravenous lines (> G18). During the procedure oxygen $(4-8 \mathrm{~L} / \mathrm{min})$ was applied via face mask or laryngeal mask, allowing sampling of exhaled carbon dioxide in non-intubated patients during administration of supplementary oxygen. Mean et $\mathrm{CO}_{2}$ values below $25 \mathrm{mmHg}$ were considered as not reliable and patient-specific $\mathrm{CO}_{2}$ values were omitted accordingly. Oxygen was delivered to maintain oxygen saturation $\left(\mathrm{SpO}_{2}\right)$ above $92 \%$. Nebulization of $3 \mathrm{~mL}$ lidocaine $2 \%$ before induction and immediately prior to surgery helps avoiding coughing due to hilar manipulation and lung traction (Flexicare Dual Mask ${ }^{\mathrm{TM}}$, Flexicare Medisize Germany, Siegburg, Germany; Aeroneb Pro $\mathrm{X}^{\mathrm{TM}}$, WKM GmbH, Emmering, Germany). When encountering excessive cough as result of surgical manipulation, additional intrathoracic vagal nerve blockade was performed with $2-5 \mathrm{~mL}$ ropivacaine $0.5 \%$. Regional anaesthesia was administered according to the planned surgical intervention (minor/major pulmonary surgery) and taking into account potential contraindications and/ or patients' requests. We used two different neuraxial regional anaesthetic approaches: TEA and PVB. TEA was adapted from departmental anaesthesiological algorithms for open thoracic surgery and encouraged in patients scheduled for major surgery or dealing with chronic pain 
syndrome. An epidural catheter was inserted between T4-T5 in sitting position. A test dose $3 \mathrm{~mL}$ bupivacain $0.5 \%$ was administered followed by $8-10 \mathrm{~mL}$ of ropivacaine $0.2 \%$ and $10 \mu \mathrm{g}$ of sufentanil. The goal was to achieve sufficient sensory and motor blockade while preserving diaphragmatic respiration. Patients received automated infusion pumps for patient-controlled epidural anaethesia (CADD ${ }^{\mathrm{TM}}$, Smiths Medical, Ashford, United Kingdom). Alternatively, ultrasound-guided PVB was performed. A blockade of several intercostal spaces is achieved using a single shot of $20 \mathrm{~mL}$ ropivacaine $0.5 \%$ at thoracic level $\mathrm{T} 5$. In minor procedures and whenever thoracic epidurals were not feasible, ultrasound-guided ESPB at T5 level with a single shot of ropivacaine $0.5 \%(20 \mathrm{~mL})$ was administered (18). Sedation was achieved by continuous infusion of dexmedetomidine $(200 \mu \mathrm{g} / 50 \mathrm{~mL})$ starting with $3 \mu \mathrm{g} / \mathrm{kg} / \mathrm{h}$ for 10 minutes and $0.5-1 \mu \mathrm{g} / \mathrm{kg} / \mathrm{h}$ until the end of surgery. If analgesia/sedation was insufficient small boli of sufentanil and propofol could be administered. We performed EEG-controlled sedation only when utilizing a lanryngeal mask and depth of sedation varied between a RASS score of 0 to -3 ("conscious sedation"). In the operating theatre patients were placed in lateral decubitus position and the lung collapsed after chest wall incision. If significant desaturation occurred, a chest tube under continuous suction provided short-term re-expansion of the collapsed lung. Patients requiring conversion to general anaesthesia were induced with propofol $(1-2 \mathrm{mg} / \mathrm{kg})$, sufentanil $(0.2 \mathrm{mg} / \mathrm{kg})$ and rocuronium $(0.5 \mathrm{mg} / \mathrm{kg})$. General anaesthesia was maintained using inhaled sevoflurane or propofol $(5 \mathrm{mg} / \mathrm{kg} / \mathrm{h})$ and rocuronium. Double lumen endotracheal tube or endotracheal tube with bronchus blocker (EZ-Blocker ${ }^{\mathrm{TM}}$ Endobronchial Blocker, 7 Fr., Teleflex Medical Europe Ltd, Athlone, Ireland; Arndt Endobronchial Blocker Set, 9 Fr., COOK MEDICAL LLC, Bloomington, IN, USA) were placed using video-laryngoscopy (C-Mac ${ }^{\mathrm{TM}}$, KARL STORZ SE \& Co. KG, Tuttlingen, Germany) or fibre-optic bronchoscopy. Fibre-optic guided intubation via laryngeal mask airway was also defined in our niVATS protocol to provide a viable bail-out. Criteria for conversion to general anaesthesia were severe hypoxaemia $\left(\mathrm{PaO}_{2}<60 \mathrm{mmHg}\right)$, severe hypercapnia with acidosis $(\mathrm{pH}<7.1)$, haemodynamic instability, persistent cough in spite of intrathoracic vagal nerve blockade, excessive diaphragmatic/mediastinal movement, failure of regional anaesthesia, incomplete iatrogenic pneumothorax, and surgical complications (major bleeding, lack of progress, adhesions, unexpected situs). All patients were scheduled to recover in the post anaesthesia care unit (PACU) after surgery prior to same-day transferal to the surgical ward.

\section{ARISCAT score}

The ARISCAT risk score (Assess Respiratory Risk in Surgical Patients in Catalonia) is a prediction score for identification of patients at risk of developing postoperative pulmonary complications (PPCs) (19). ARISCAT is composed of seven preoperative patient characteristics (age, comorbidities/respiratory infection, $\mathrm{SpO}_{2}$, anemia, type/ site of surgery, operative times, emergency). The resulting risk score intervals are defined as: low risk $<26$ points $(1.6 \%$ risk of developing PPCs), Intermediate Risk 26-44 points (13.3\% risk of developing PPCs) and high risk $\geq 45$ points (42.1\% risk of developing PPCs).

\section{LAS VEGAS score}

The LAS VEGAS risk score (Local Assessment of Ventilatory Management During General Anaesthesia for Surgery) based on 13 perioperative items predicts PPCs (20). Additionally to ARISCAT, LAS VEGAS includes intraoperative events. Risk levels are defined as: low risk $\leq 7$ points (2\% risk of developing PPCs), moderate risk 8-16 points ( $8 \%$ risk of developing PPCs), high risk $\geq 17$ points (22\% risk of developing PPCs).

\section{ThRCRI}

Thoracic revised cardiac risk index (ThRCRI) score was developed to predict risks of postoperative major cardiac complications in thoracic surgery (21). It comprises four weighted predictors: ischaemic heart disease (scoring 1.5 points); history of cerebrovascular disease (1.5 points); serum creatinine level greater than $2 \mathrm{mg} / \mathrm{dL}$ (1 point) and pneumonectomy (1.5 points). The patients are grouped into four classes (Risk class/predicted major cardiac complications: $\mathrm{A} / 1.5 \%$; $\mathrm{B} / 5.8 \%, \mathrm{C} / 19 \%, \mathrm{D} / 23 \%$ ).

\section{Statistical analysis}

Explorative and descriptive data analysis of relevant variables (patient characteristics, intraoperative data, and postoperative findings) was performed, and data was presented as mean $(\mathrm{M}) \pm \mathrm{SD}$ and/or $95 \% \mathrm{CI}$ (confidence intervals) or as percentage/frequency of categorical 
variables. Imputations methods to handle missing data were not adopted. Missing values are indicated in tables and legends. Komolgorov-Smirnov test (KS test) was used to determine normal distribution. Levene's test was used to assess equality of variances for a variable and common $t$-test for unpaired variables (KS $\mathrm{P}>0.05$ ) or non-parametric Mann-Whitney-U test $(\mathrm{KS} \mathrm{P}<0.05)$ was used to determine if there were differences in variables between major and minor surgery groups. Pearson's chi-squared test $\left(\chi^{2}\right)$ or Fisher's exact test (if expected cell frequencies were below 5) was conducted to determine statistically significant differences between expected frequencies and observed frequencies in one or more categorical categories. Level of significance is defined as $\mathrm{P}<0.05$ (two-sided). Data analysis was performed with GraphPad Prism 5 (GraphPad Software, Inc., San Diego, CA, USA, RRID:SCR_002798) and IBM SPSS Statistics (Version 26.0, IBM Corp., Armonk, NY, USA, RRID:SCR_002865).

\section{Results}

Between June 2018 and December 2019, a total of $n=88$ consecutive patients underwent niVATS for minor or major thoracic procedures. Major resections included lobectomies $(\mathrm{n}=25)$ and segmentectomies $(\mathrm{n}=15)$; minor procedures included wedge resections $(n=32)$, pleurodesis $(n=8)$, empyema debridement/decortication $(n=4)$, biopsies $(n=2)$ and haematoma evacuation $(n=2)$. Patients characteristics in minor and major surgery did differ significantly in age $(60.14 \pm 17.42$ vs. $67.94 \pm 12.28$ years, $\mathrm{P}=0.038)$, height (1.74 \pm 0.09 vs. $1.70 \pm 0.08 \mathrm{~cm}, \mathrm{P}=0.015)$, weight $(78.62 \pm 17.96$ vs. $69.55 \pm 15.89 \mathrm{~kg}, \mathrm{P}=0.015)$, ASA classification $(\mathrm{P}=0.014)$, expected FEV1 $(3.05 \pm 0.76$ vs. $2.71 \pm 0.67 \mathrm{~L}, \mathrm{P}=0.041)$, expected FVC $(3.97 \pm 0.93$ vs. $3.46 \pm 0.85 \mathrm{~L}, \mathrm{P}=0.018)$, Tiffeneau test $(72.95 \% \pm 12.83 \%$ vs. $62.91 \% \pm 17.26 \%$, $\mathrm{P}=0.006)$, rate of preoperative respiratory infection $(\mathrm{P}=0.003)$ and preoperative creatinine level $(99.85 \pm 76.94$ vs. $77.50 \pm 19.97 \mathrm{mg} / \mathrm{dL}, \mathrm{P}=0.040$ ) (Table 1).

Intraoperative respiratory parameters did not differ significantly between minor and major surgery groups, except for mean respiratory rate $(\mathrm{P}<0.001)$ and mean etCO $(\mathrm{P}<0.001)$. Mean oxygenation $\left(\mathrm{SpO}_{2}\right)$ was similar between both groups (mean $\mathrm{SpO}_{2}$ minor group: $95.72 \% \pm 2.46 \%$, major group: $96.11 \% \pm 2.20 \% ; \mathrm{P}=0.442$ ), whereas mean etCO $\mathrm{C}_{2}$ differed significantly without any clinical relevance (minor group: $37.56 \pm 7.31 \mathrm{mmHg}$, major group: $48.42 \pm 10.09 \mathrm{mmHg}, \mathrm{P}<0.001)$. Lowest $\mathrm{SpO}_{2}$ did not differ between minor and major surgery groups $(90.52 \% \pm 5.79 \%$ vs. $89.00 \% \pm 7.94 \%, \mathrm{P}=0.303$ ), whereas highest etCO $\mathrm{CO}_{2}$ did $(44.78 \pm 11.63$ vs. $65.39 \pm 16.58, \mathrm{P}<0.001)$ with mean highest $\mathrm{CO}_{2}$ values $20.61 \mathrm{mmHg}$ (95\% CI: 12.96, 28.62) lower for the minor surgery group (Figure 2). Utilization of laryngeal mask was allowed for both groups, and was implemented more often in major resection $(22.9 \%$ vs. $62.5 \%, \mathrm{P}<0.001)$. Need for conversion to conventional endotracheal intubation did not differ between groups $(\mathrm{P}=0.088)$. In line with departmental protocols, TEA was performed more frequently in major surgery $(18.8 \%$ vs. $70.0 \%, \mathrm{P}<0.001)$, whereas ESPB were performed more often in minor procedures $(20.8 \%$ vs. $2.5 \%, \mathrm{P}<0.001)$. Use of sufentanil was significantly higher in major surgery patients $(11.20 \pm 8.14$ vs. $20.10 \pm 19.76 \mu \mathrm{g}, \mathrm{P}=0.010)$, as was intraoperative vagal nerve blockade $(33.3 \%$ vs. $95 \%, \mathrm{P}<0.001 ; 0$ vs. $17.5 \%$, $\mathrm{P}=0.003)$. Operation duration was significantly longer in major surgery $(35.96 \pm 17.02$ vs. $124.05 \pm 74.49 \mathrm{~min}, \mathrm{P}<0.001)$, with mean times 88.09 minutes (95\% CI: 63.82, 112.37) lower for the minor surgery group. Accordingly, total fluid administration was significantly higher in major surgery $(528.13 \pm 249.45$ vs. $963.75 \pm 578.46 \mathrm{~mL}, \mathrm{P}<0.001)$. The use of catecholamines $(<0.04 \mu \mathrm{g} / \mathrm{kg} / \mathrm{min})$ was significantly higher in major resections (6.3\% vs. $45 \%, \mathrm{P}<0.001)$ (Table 2).

Postoperative findings including pain score on numeric rating scale (NRS), use of opiods, application of oxygen, admission to ICU did not differ significantly between both groups, except for mean chest tube duration $(3.34 \pm 2.67 \mathrm{vs}$. $6.93 \pm 5.71$ days, $\mathrm{P}<0.001)$ with mean chest tube duration 3.58 days (95\% CI: $1.67,5.49)$ lower for the minor surgery group. Mean postoperative hospital stay differed significantly between groups $(6.40 \pm 4.51 v s .10 .10 \pm 6.50$ days, $\mathrm{P}<0.001$ ) (Table 3).

Overall morbidity was $28.4 \%$. No perioperative death was encountered, and 30-day mortality was $0 \%$. Postoperative complications are listed in Table 4. A persistent air leak (air leakage $>5$ days) occurred in $4.2 \%$ of minor procedures and $20 \%$ of major procedures $(\mathrm{P}=0.039)$. PPCs like pneumothorax, bronchospasm, aspiration, atelectasis, wound infection, postoperative pulmonary infection, or respiratory failure did not differ between groups. Overall PPC rate was $15.9 \%$. ARISCAT differed significantly between minor and major surgery groups $(39.92 \pm 13.84$ vs. $48.15 \pm 12.72, \mathrm{P}=0.002)$. These values correspond to an intermediate risk for PPCs (13.3\% expressed in numbers) in minor surgery patients and a high risk for PPCs $(42.1 \%$ expressed in numbers) in major surgery patients. LAS VEGAS differed significantly between minor and major surgery $(14.83 \pm 7.57$ vs. $26.05 \pm 8.28, \mathrm{P}<0.001)$. These values 
Table 1 Characteristics of patients according to minor and major surgery

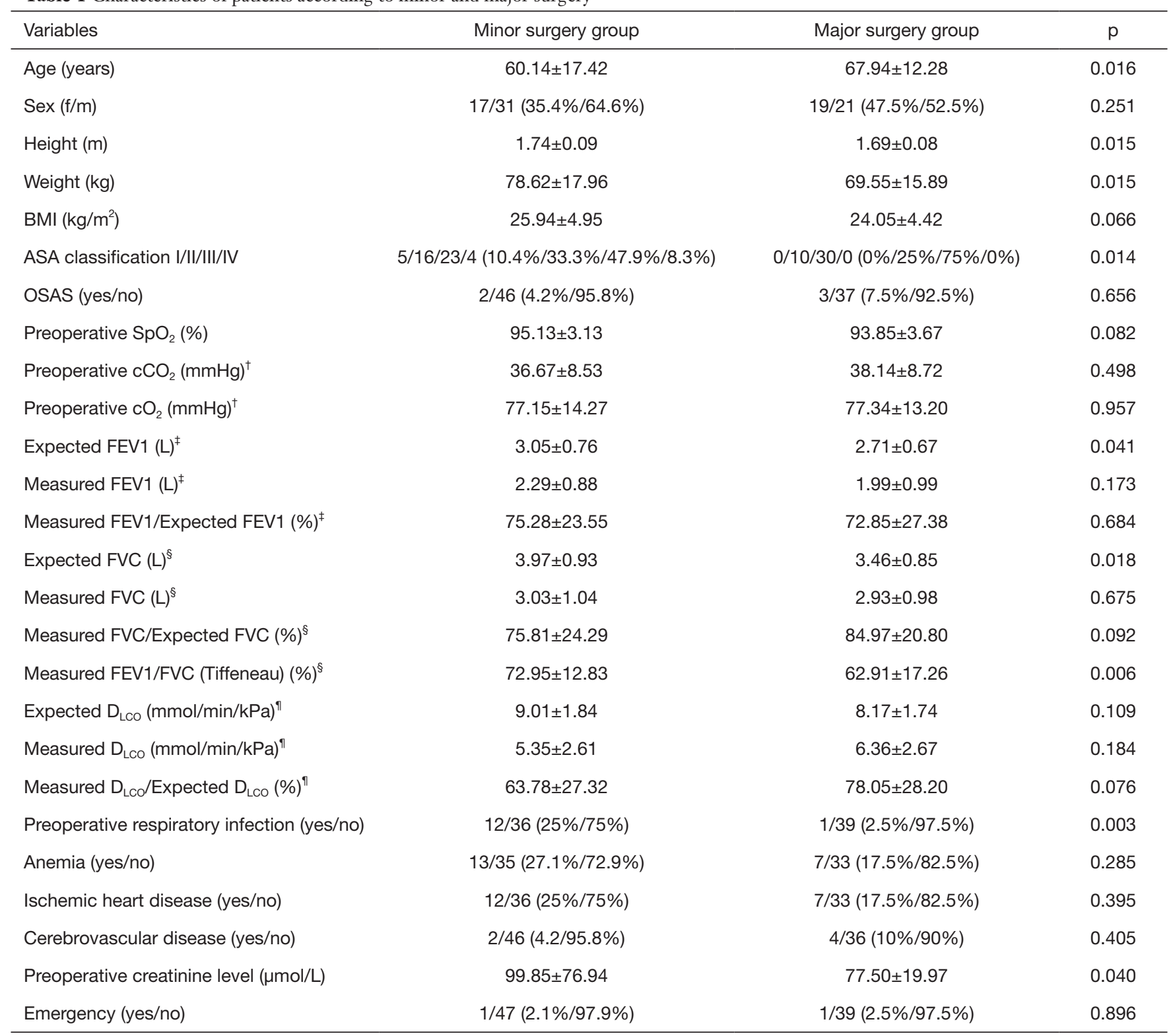

Continuous data are presented as mean \pm standard deviation (SD) and categoric variables as number (frequency and/or \%). $\mathrm{P}<0.05$ is considered significant. In terms of lung function tests and capillary blood gas analysis some values could not have been stored for the observed variable because of invalid results (e.g., pneumothorax), underlying diseases, failed documentation, or lack of indication. ${ }^{\dagger}$, minor $n=33$, major $n=31$; ${ }^{\ddagger}$, minor $n=40$, major $n=34 ;{ }^{\S}$, minor $n=40$, major $n=33$; ${ }^{n}$, minor $n=32$, major $n=20$. BMI, body mass index; ASA, American Society of Anesthesiology; OSAS, obstructive sleep apnoea syndrome; $\mathrm{SpO}_{2}$, peripheral oxygen saturation; $\mathrm{cCO}_{2}$, capillary carbon dioxide partial pressure; $\mathrm{CO}_{2}$, capillary oxygen partial pressure; FEV1, forced expiratory volume in one second; FVC, forced vital capacity; $\mathrm{D}_{\mathrm{LCO}}$, diffusing capacity of the lung for carbon monoxide.

correspond to moderate risk for PPCs ( $8 \%$ expressed in numbers) in minor surgery and a high risk (22\% expressed in numbers) in major surgery. The overall incidence of cardiac complications was about $1.1 \%(\mathrm{n}=1$ acute coronary syndrome during minor surgery). Patients were assigned to risk classes according to their ThRCRI score: class A and B (defined as "low risk") and class C and D (defined as "high risk"). There were no significant differences between minor and major surgery groups $(\mathrm{P}=0.371)$, except for more class C patients in minor surgery (Table 4). 

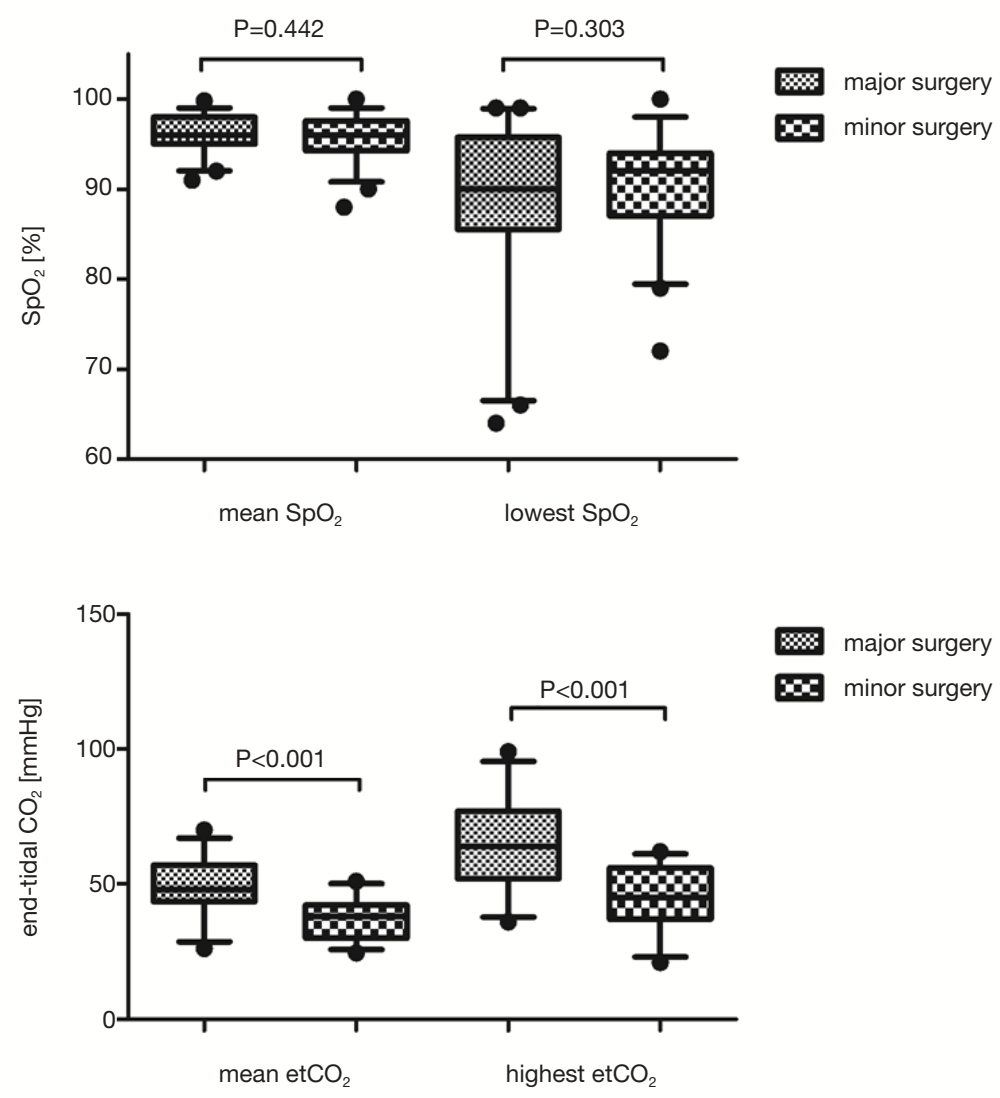

Figure $2 \mathrm{SpO}_{2}$ and end-tidal $\mathrm{CO}_{2}$ in minor and major surgery group. $\mathrm{SpO}_{2}$ (upper panel) and end-tidal $\mathrm{CO}_{2}$ (lower panel) in minor and major surgery group. Data are presented as mean \pm standard deviation (box) and 95\% CI (whiskers), black dots represent outliers. SpO ${ }_{2}$, peripheral oxygen saturation; et $\mathrm{CO}_{2}$, end-tidal carbon dioxide partial pressure, $\mathrm{P}<0.05$ is considered significant. $\mathrm{A}$ few etCO values were missing due to failed documentation or invalid etCO $\mathrm{CO}_{2}$ monitoring. Mean etCO $\mathrm{C}_{2}$ values below $25 \mathrm{mmHg}$ were considered as not reliable and patient-specific $\mathrm{CO}_{2}$ values were omitted accordingly (minor $\mathrm{n}=27$, major $\mathrm{n}=31$ ).

\section{Discussion}

Primary end point of our study was to evaluate feasibility and safety of a minimally invasive anaesthesiological approach in niVATS in minor and major pulmonary resection after first introducing the technique at our institution in 2018 (22). Both groups had no early mortality and low conversion and complication rates, suggesting a general viability to our approach. Demographics in both groups were similar, with a trend towards more obstructive disease in the major surgery group and a higher count of ASA IV-classified patients in the minor surgery group.

As was to be expected due to longer average operating times, higher maximum and mean end-tidal $\mathrm{CO}_{2}$ values were found in patients undergoing major surgery, with no differences in oxygenation. This did not lead to relevant hemodynamic instability as sign of acute right heart failure, and correlates well with findings of other working groups that described similar $\mathrm{CO}_{2}$ values in arterial blood gas samples without any hemodynamic consequences. There is evidence that awake or sedated patients with normal right ventricular function and $\mathrm{PaCO}_{2}$ levels up to $70 \mathrm{mmHg}$ are likely tolerate it well (23).

In contrast, the respiratory rate was higher in the minor surgery group, with a lower use of opioids to modulate it. In minor and thus likely shorter interventions (pleural, lung or mediastinal biopsies, resection of peripheral nodules, pneumothorax, empyema), experienced surgeons tend to accept a higher respiratory rate, as it is less likely to affect surgical outcome.

In accordance with our protocol, TEA was regularly implemented in major resection, which may be one reason 
Table 2 Intraoperative measures and procedures

\begin{tabular}{|c|c|c|c|}
\hline Variables & Minor surgery group & Major surgery group & $P$ \\
\hline Respiratory rate highest $\left(\mathrm{min}^{-1}\right)^{\dagger}$ & $25.82 \pm 6.86$ & $20.28 \pm 6.04$ & $<0.001$ \\
\hline Respiratory rate lowest $\left(\min ^{-1}\right)^{\dagger}$ & $10.61 \pm 4.71$ & $6.10 \pm 3.58$ & $<0.001$ \\
\hline $\mathrm{SpO}_{2}$ mean $(\%)$ & $95.72 \pm 2.46$ & $96.11 \pm 2.20$ & 0.442 \\
\hline Laryngeal mask (yes/no) & $11 / 37(22.9 \% / 77.1 \%)$ & $25 / 15(62.5 \% / 37.5 \%)$ & $<0.001$ \\
\hline TEA (yes/no) & 9/39 (18.8\%/81.3\%) & 28/12 (70\%/30\%) & $<0.001$ \\
\hline PVB (yes/no) & 15/33 (31.3\%/68.8\%) & 19/21 (47.5\%/52.5\%) & 0.119 \\
\hline ESPB (yes/no) & 10/38 (20.8\%/79.2\%) & $1 / 39(2.5 \% / 97.5 \%)$ & 0.010 \\
\hline Tranexamic acid (yes/no) & $3 / 45$ (6.3\%/93.8\%) & 7/33 (17.5\%/82.5\%) & 0.175 \\
\hline Transfusion (yes/no) & $0 / 48(0 \% / 100 \%)$ & 0/40 (0\%/100\%) & 1.000 \\
\hline Fluid administration (mL) & $528.13 \pm 249.45$ & $963.75 \pm 578.46$ & $<0.001$ \\
\hline Catecholamins (yes/no) & 3/45 (6.3\%/93.7\%) & $18 / 22(45 \% / 55 \%)$ & $<0.001$ \\
\hline Conversion (yes/no) & $1 / 47(2.1 \% / 97.9 \%)$ & $5 / 35(12.5 \% / 87.5 \%)$ & 0.088 \\
\hline Operative time (min) & $35.96 \pm 17.02$ & $124.05 \pm 74.49$ & $<0.001$ \\
\hline
\end{tabular}

Table 3 Postoperative measures in post-anaesthesia care units (PACU, ICU, normal ward)

\begin{tabular}{|c|c|c|c|}
\hline Variables & Minor surgery group & Major surgery group & $\mathrm{P}$ \\
\hline Opioid use PACU (yes/no) & $12 / 36(25 \% / 75 \%)$ & $10 / 30(25 \% / 75 \%)$ & 1.000 \\
\hline $\mathrm{O}_{2}$ application (yes/no) & $19 / 29(39.6 \% / 60.4 \%)$ & $16 / 24(40 \% / 60 \%)$ & 0.968 \\
\hline Chest tube duration $(d)^{\S}$ & $3.34 \pm 2.67$ & $6.93 \pm 5.71$ & $<0.001$ \\
\hline Hospital LOS (days) ${ }^{\pi}$ & $6.40 \pm 4.51$ & $10.10 \pm 6.50$ & $<0.001$ \\
\hline
\end{tabular}

Continuous data are presented as mean \pm standard deviation (SD) and categoric variables as number (frequency and/or\%). $\mathrm{P}<0.05$ is considered significant. Missing NRS data $(n=3)$ occurred due to failed documentation. Supplemental oxygen was not documented in two cases, because two evaluated patients were not admitted to PACU postoperatively. Chest tube management was not standardized in patients outside the department of thoracic surgery. In a few cases $(n=6)$ hospital length of stay was determined by the (thoracic-nonspecific) underlying disease and was not related to the particular thoracic operation. ${ }^{\dagger}$, minor $n=46$, major $n=39$; ${ }^{\ddagger}$, minor $n=47$, major $n=39$; ${ }^{\S}$, minor $n=44$, major $n=40$; ", minor $n=43$, major $n=39$ ). NRS, numeric rating scale; PACU, post-anaesthesia care unit; $O_{2}$, oxygen; LOS, length of stay. 
Table 4 Postoperative complications

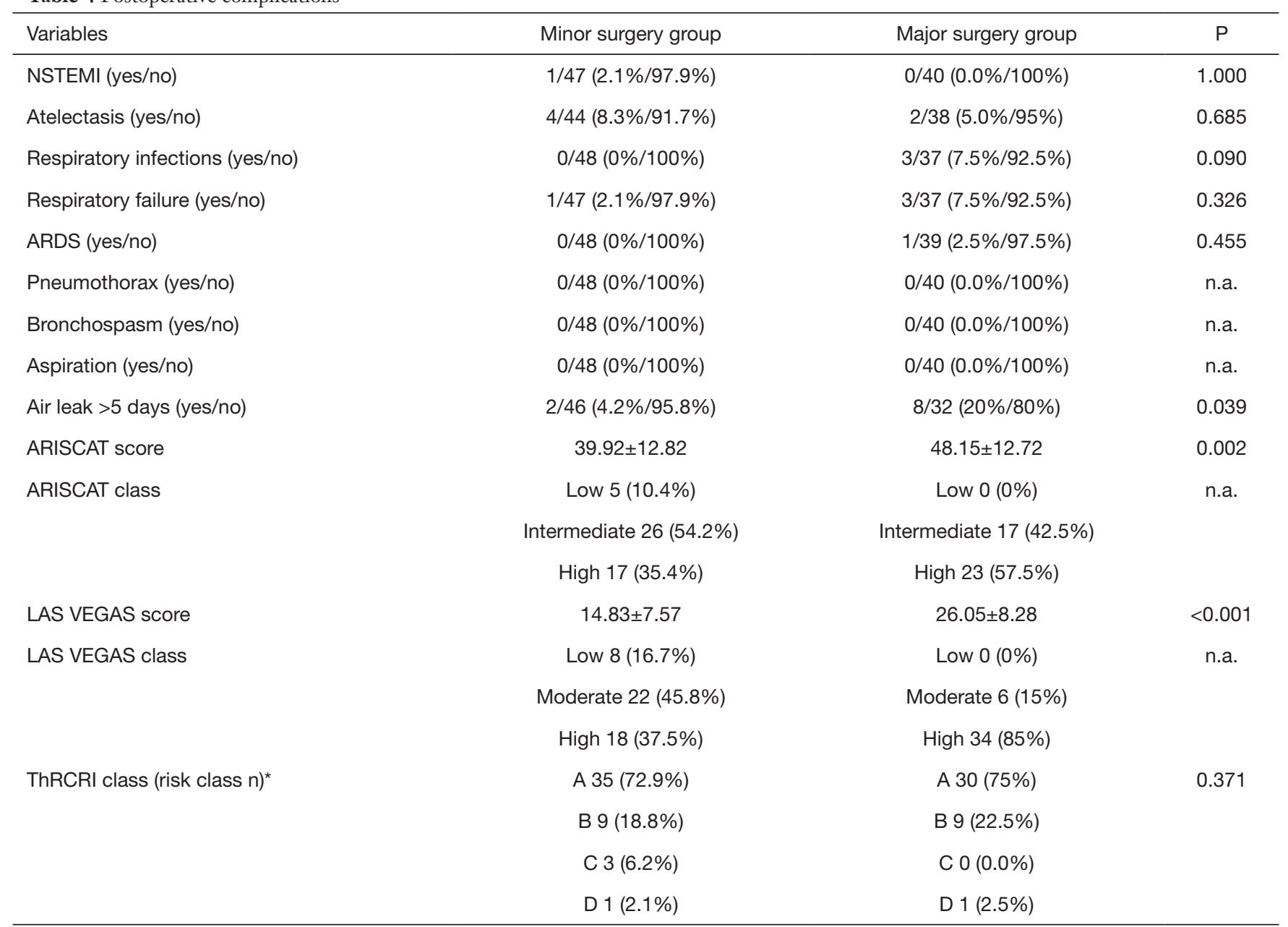

Continuous data are presented as mean \pm standard deviation (SD) and categoric variables as number (frequency and/or\%). $\mathrm{P}<0.05$ is considered significant. *, A, B: "low risk"; C, D: "high risk". LAS VEGAS, Local Assessment of Ventilatory Management During General Anaesthesia for Surgery and effects on Postoperative Pulmonary Complications; ARISCAT, Assess Respiratory Risk in Surgical Patients in Catalonia; ThRCRI, Thoracic Revised Cardiac Risk Index; n.a., not applicable.

for a more frequent use of catecholamines in this group. The dose never exceeded $0.1 \mu \mathrm{g} / \mathrm{kg} / \mathrm{min}$ and could always be stopped at the end of the operation. Based on body weight and infusion rate, fluid adminsitraton exceeded $6 \mathrm{~mL} / \mathrm{kg} / \mathrm{h}$ in minor surgery. Considering a retrospective analysis of the German Thorax Registry, $>6 \mathrm{~mL} / \mathrm{kg} / \mathrm{h}$ of crystalloids, the FEV1, operative time and preoperative $\mathrm{PaO}_{2}$ were found to be independent risk factors for occurrence of PPCs (16). In contrast, $\mathrm{Wu}$ et al. showed an increased incidence of postoperative complications with infusion rates $<9.4$ or $>11.8 \mathrm{~mL} / \mathrm{kg} / \mathrm{h}$ during minimally invasive lobectomies (24).

Especially during our initial learning curve, laryngeal masks were used regularly in major resection. Apart from keeping the patients' airway open during anaesthesia, they provide a higher inspiratory oxygen concentration and more robust and reliable end-tidal $\mathrm{CO}_{2}$ signal but require deeper sedation. At present no published evidence comparing laryngeal mask and oxygen mask in niVATS exists (25). Our overall conversion rate to orotracheal intubation was $6.8 \%$ and did not differ significantly between both groups, which is in line with data published by other groups who reported conversion rates between $2-10 \%$ depending on the experience of the centre and the extent of the intervention (minor vs. major surgery) $(26,27)$. Reasons for conversion were excessive breathing excursions $(n=3)$, exceedingly long operatinge time $(n=2)$ and intraoperative seizure $(n=1)$, 
which mirrors previously published data $(26,28,29)$. A total of three bleedings from the pulmonary artery (blood loss $<500 \mathrm{~mL}$, no transfusion trigger) could be managed via VATS under spontaneous breathing. To ensure patient safety we encourage an algorithm for emergent intubation and lung separation to be established prior to surgery, as intubation in lateral decubitus position is challenging.

While less invasive approaches have been considered standard of care in thoracic surgery for over a decade (3), anaestesiological concepts are often still rooted in the late 1990ies. In most thoracic units, central iv lines, arterial blood pressure measurement and urinary catheters, among other, are commonplace for most procedures, and postoperative care is often administered on dedicated ICUs $(30,31)$. Minimally invasive surgery such as uniportal VATS has seen significant advances in recent years. Hence, there is a paradigm shift in anaesthesia towards more minimally invasive approaches to improve the risk/benefit profile of analgesic and sedative techniques. In our opinion, the move towards "minimally invasive anaesthesia", e.g., non-intubated VATS, parallels similar endeavours in uniportal VATS. The primary focus of our pathway is to avoid mechanical ventilation, invasive blood pressure measurements, central venous lines, monitoring in intensive care units etc. and to implement modern analgesic-sedative concepts like the use of dexmedetomidine combined with (ultrasound-guided) regional anaesthesia. In the long term (of course after completion of the learning curve), the focus is on faster recovery after surgery and less sideeffects attributed to general anaesthesia and mechanical ventilation/one-lung ventilation. Unfortunately, we wanted to become as "minimally invasive" as possible by tweaking an established but distressing anaesthetic procedure (e.g., invasive monitoring, mechanical ventilation, bladder catheterization). Due to the fact that we present a retrospective data collection and observational study design, a robust comparison between our (new) pathway and other (conventional) approaches would not even be possible. NiVATS takes a different approach, relies on adequate pain relief and sedation, enabling even complex thoracic procedures under spontaneous breathing. At this point no anaesthesiological gold standard for niVATS does exist, and these procedures are often limited to dedicated centres $(25,26,28)$. We postulate that VATS paired with a concomittant minimally invasive anesthesiological concept may result in less perioperative morbidity and improved outcome.

Postoperative chest tube duration and hospital LOS after major surgery exceeded times previously published (26), which prompted us to modify our chest tube management protocols (32) gearing it towards an ERAS program more in line with current international guidelines (33). A persistent air leak occurred in $11.4 \%$ of total cases, which corresponds to data previously published by other groups that reported an incidence between 6-13\% (34). Our comparatively long chest tube duration and the ratio of examined air leaks might be related to the fact that our major resection group included several anatomical resections in lung volume reduction procedures. These are known to be associated with longer chest tube duration and are technically challenging. Basically, surgical technique does not differ from uniportal VATS procedures under general anaesthesia, but certainly requires a higher degree of surgical skills (mediastinal movement, diaphragmatic motion, initial cough reflex etc.) and an increased awareness of the patient with "conscious sedation". We believe that upon completion of an initial learning curve, associated metrics like air leaks and chest tube duration will improve. Furthermore, in nonintubated VATS residual air leak is harder to detect than under mechanical ventilation.

\section{Anaesthesiological management}

Utilizing anaesthetics and opioids for sedation in combination with supraglottic airway devices to improve ventilation and prevent airway obstruction is defined as monitored anaesthesia care (MAC) (26) and was our initial choice for major lung surgery. We further recommend TEA as an adjunct for adequate and repeatable analgesia in these cases, as it is a known mainstay of both niVATS and conventional lung surgery (9). Ultrasound-guided PVB was selected in shorter operations and seems to offer comparable analgesia to TEA with fewer side effects (35). If this is not feasible, usually under running anticoagulant therapy, ultrasound-guided ESPB or an intraoperative intercostal blockade (IB) are encouraged (18). In any case, surgeons should always perform an incisional infiltration with local anesthetics to bridge the onset of peripheral or neuraxial regional anaesthesia.

Optimal conditions for major pulmonary surgery were usually obtained by combining neuraxial regional anaesthesia with hypnotic drugs (dexmedetomidine +/propofol) and opioids (sufentanil boli), as an epidural anaesthesia does not block neither the vagal nor the phrenic nerve, which are responsible for the cough reflex. This stresses the need for intrathoracic vagal blockade, which we 
performed in almost all major and a third of minor cases, especially when manipulating the bronchial tree. In our personal experience almost no coughing occurs regardless of surgical manipulation after successfully performing a vagal blockade, which has been reported by other groups as well (36).

Dexmedetomidine was used for sedation in both groups and it's reported advantages include prolongation of local anesthetics effects $(37,38)$ and potentially a lower risk for upper airway collapsibility compared to other sedatives (39). We did not observe any episode of apnea or respiratory problems linked to sedation with dexmedetomidine. It provides sedation, anxiolysis, analgesia and stress response attenuation without being inferior to other sedative substances $(38,40)$. We used a loading dose of dexmedetomidine followed by continuous infusion of dexmedetomidine in order to avoid excessive sedation that could lead to airway problems or haemodynamic impairments. In our opinion, dexmedetomine is a suitable sedative for non-intubated VATS. We suggest performing sedation with dexmedetomidine in combination with regional anaesthetic approaches which is in concordance with other approaches (41). In our patients we performed non-intubated VATS using dexmedetomidine and TEA/ PVB or ESPB. We achieved sufficient sedation and analgesia in majority of cases. If analgesia/sedation was insufficient small boli of sufentanil and propofol could be administered (Table 2). The average amount of the used opioid (sufentanil) used was only $15.2 \mu \mathrm{g}$. None of the conversions occurred due to poor haemodynamics or overdosage of additive sedatives or opioids.

A gold standard of anaesthetic technique should not be obtained. Previous studies showed that different anaesthetic approaches are feasible $(9,42,43)$. However, a direct comparison of the approaches seems difficult, as most of the original papers and most of the reviews give little information on the regimes and exact dosages of sedatives used (27). Furthermore, our anaesthetic technique based on sedation with dexmedetomidine could not be directly compared with other published techniques based on propofol and remifentanil, midazolam and/or sevoflurane due to statistical flaws (lack of control group).

The sedatives used do not seem to generate any relevant differences in clinical practice. Data published from other centres show essentially similar conversion rates and similar frequencies of the need for mild catecholamine therapy. Apnoeas forcing conversion are rare with both propofol and dexmedetomidine $(26,28,29)$. Nevertheless, incorrect dosing of sedatives and opioids may lead to respiratory depression and sedation. Drugs with less effect on respiration and consciousness are preferred.

Our aim was to keep anaesthesiological monitoring to a minimum and further reduce the overall invasiveness of the procedure. No central venous catheters, no arterial lines for invasive blood pressure measurements and no urinary catheters were placed. Our low conversion and complication rate seem to justify this approach, and truly tubeless procedures may be feasible in the forseable future. Postoperative avoidance of ICU was achieved in $94.4 \%$ of all cases and most patients were mobilized on the day of surgery.

The postoperative low pain intensity can easily be explained by consistent use of regional anaesthesia procedures according to the standard of care (Figure 2) and nicely translates from conventional VATS for both PVB and TEA (35).

\section{Procedure-related complications and risk classification}

The overall incidence of PPCs following thoracic surgery varies primarily due to the type of pulmonary complications, the clinical criteria used for PPC definition and the type of surgery (major/minor surgery). There are various scores for predicting postoperative pulmonary complications, including ARISCAT and LAS VEGAS $(19,20)$. ARISCAT was developed and validated in 2010 and achieves the best prediction results in Western Europe (19). The newer LAS VEGAS score focuses on intraoperative events to predict occurrence of PPCs (20) and takes the effect of supraglottic airway devices and spontaneous breathing without muscle relaxation into account. In our study, the mean ARISCAT score in minor surgery was 39.92 points, predicting an intermediate risk of PPCs, which means that the predicted frequency of PPCs was $13.3 \%$. The true incidence of PPCs in minor procedures was $10.4 \%$. The patients scheduled for major surgery had a high calculated risk of developing PPCs (ARISCAT score 48.15 points), i.e., predicting PPCs in $42.1 \%$ of patients, but true incidence of PPCs was just $22.5 \%$.

The mean LAS VEGAS score in minor surgery was 14.83 points and 26.05 points in major surgery. Accordingly, the predicted risk of PPCs in minor surgery is moderate (prediction of PPCs in $8 \%$ ) and high in major surgery (prediction of PPCs in $22 \%$ ). It reflected the true postoperative complication rate in our groups much more closely. One reason for this could be that the score also 
includes intraoperative parameters, such as invasiveness of ventilation, type of anesthesia and intubation or use of a supraglottic airway device.

Although risk prediction equations for postoperative postoperative complications derived from ARSICAT and LASVEGAS score permit general estimates of risk in patients undergoing a variety of surgical procedures, they do not include data from the physical examination or pulmonary function tests, which are also employed by clinicians to assist in risk prediction before thoracic surgery. Furthermore, the ARISCAT/LASVEGAS derivation cohort had only $1.4 \% / 2.8 \%$ of its patients undergoing thoracic surgery. This underrepresented sample can be seen as a limitation using these scores.

However, producing a risk prediction formula for thoracic surgeries seems to be difficult. Lung resection procedures and lung manipulations or iatrogenic pneumothorax result in physiology deficit. This deficit is variable according to the extent and duration of the surgical procedure and of course the pre-existing lung function.

Predicted postoperative FEV1 (or even DLCO) would be better for preoperative evaluation and clinical risk assessment, but retrospective data are limited. Predicting postoperative complications in thoracic surgery remains an arduous task. Examined patients presented as a very heterogeneous thoracic-surgical population, diverging severity of underlying and secondary diseases, often requiring complex procedures, with an outcome often hard to predict. When we decided to use the present scores, we tried to take into account the results of other, more specific previous studies, combining pulmonary function associated parameters with items such as age, ASA status, $\mathrm{BMI}, \mathrm{SpO}_{2}$, COPD, smoking history, preoperative activity, congestive heart failure, surgery duration, etc., as they are related to occurrence of postoperative pulmonary complications $(16,44,45)$. In addition, definitions of PPC are often not explicit and differ among studies. Thus, we felt compelled to use scores which represent the lowest common denominator for our patient cohort. In our opinion, our risk assessment for minor and major surgery patients based on a score like ARISCAT and LASVEGAS reflects a standardized estimation of the predicted postoperative risk, which can be interpreted by trend. Of course, these scores cannot predict which individual thoracic patient may develop complications.

As there is no consensus on the best PPC score to use, an alternative and certainly good solution would be the European Society Objective Score (ESOS) applied by
Brunelli. However, this was tested especially for lobectomy and pneumectomy in neoplasia (46).

In addition to PPCs, cardiac complications have to be expected in an ageing and morbid patient population. ThRCRI is used to predict these complications, especially for thoracic surgery (21). In our entire study population, the incidence of cardiac complications was about $1.1 \%$ ( $\mathrm{n}=1$ acute coronary syndrome in minor surgery group), which is well below the calculated incidence of $3.3 \%$ in the original paper of Brunelli et al. (21). This result could also indicate that right ventricular load due to higher endtidal $\mathrm{CO}_{2}$ values is not relevant. The low incidence of cardiac complications also supports the use of non-invasive monitoring.

Irrespective of patients risk classification previous data supported important clinical advantages to the patients. A small number of randomized controlled trials $(9,47)$ and two recent meta-analyses $(48,49)$ have shown that niVATS can be associated with some advantages over an intubated technique and may be a beneficial alternative. In particular, these meta-analyses show a reduction in operating room time, a reduction in hospital length of stay and a decrease in perioperative complications $(48,49)$. In the largest RCT to date by Liu et al., postoperative morbidity was significantly lower in the non-intubated group $(\mathrm{P}=0.004)$. In particular, respiratory complications were reduced from $10 \%$ to $4.2 \%$ $(\mathrm{P}=0.039)$ (9). Other benefits have been shown including shortened recovery (47) and faster return to oral intake (9). Moreover, studies have also reported improved patient satisfaction with a non-intubated VATS approach (4).

In our opinion, it cannot be deemed appropriate to make these advantages available to all patients. So, we would argue for wider acceptance of niVATS approaches and adoption of our/any analgesic-sedative approach.

\section{Minor vs. major surgery}

These two groups are very difficult to compare due to heterogeneous patient cohort with both different underlying diseases and pathologies and surgical procedures (minor/major surgery). The application of our departmental anaesthetic pathway represents one "intervention". The surgical procedure, i.e., minor or major thoracic surgery represents a main characteristic allowing the reader to differentiate between two surgical groups. Of course, numerous metrics (e.g., fluid administration etc.) differed between groups because they are intrinsically linked to duration and extent of the procedure. Thus, the 
comparison focuses on the feasibility and practicability of our anaesthetic approach in terms of two different surgical procedures.

Furthermore, in previous studies most of the centres attempting this pathway have largely focused on minor non lung resectional procedures. Only a very limited amount of publications report larger cohorts of major resections, most of them stemming from Jin-Shing Chen's group. While minor niVATS procedures can nowadays be considered standard of care in dedicated units, the same is not true for major resections. These facts prompted us to present our personal experience in a European tertiary care centre.

Conventional VATS under general anaesthesia may have deleterious effects in some patients: side-effects of analgesics, need for intubation, diaphragm relaxation, side effects of mechanical ventilation i.e., barotrauma, volutrauma, atelectrauma and proinflammatory mediator release, increasing morbidity and mortality (26,50-52). These facts lead to an initial push in both the surgical and anaesthesiologic community, encouraging non-intubated VATS primarly in minor thoracic procedures. In our study we focus on the anaesthetic pathway and its transformation to non-invasiveness in minor but also in major surgery. We would like to describe that this anaesthetic pathway, using a standardised protocol, is equally suitable for major surgery. We concluded that the approach is safe and feasible both in minor and major surgery.

Nevertheless, there is still room for anaesthetic and surgical improvement. Furthermore, there is a high need for a larger number of prospective randomized studies that specifically compare the non-intubated VATS approach with conventional VATS for the surgical treatment of different thoracic diseases.

\section{Limitations}

Most limitations of this study are inherent to its retrospective design, e.g., presence of missing data (which had no significant effect on the conclusions that can be drawn from the data). It is a single-center study and a selection bias cannot be ruled out for our intervention groups. In addition, patients of variable ages and with various underlying and previous illnesses were included in this study. This results in heterogenous patient cohorts. Even though the PPC prediction scores predicted moderate to high risk of pulmonary complications after surgery in some patients, a large proportion of patients do not belong to the high-risk group from a clinical point of view. Finally, it is not possible to deduce whether the previously published advantages of niVATS are reproducible in our collective due to lack of a control group.

\section{Conclusions}

We encounterd a lower morbidity and mortality rate than usually reported in these patient collectives, which was even lower than risk calculation models would predict. Maintenance of spontaneous breathing without use of muscle relaxants and consistent use of regional anesthetic procedures seems to be advantageous for some patients. Foregoing central iv lines, arterial pressure measurement and urinary catheterization was reasonable, suggesting that less could be more. This prompts us to consider our niVATS protocol a viable alternative for both minor and major thoracic procedures in selected patients. Standardized holistic algorithms as well as an experienced and skilled multidisciplinary and multiprofessional team are mandatory. Randomised controlled trials comparing the presented approach with conventional approaches focusing on patientcentred outcome parameters are necessary in future.

\section{Acknowledgments}

Funding: None.

\section{Footnote}

Reporting Checklist: The authors have completed the STROBE reporting checklist. Available at http://dx.doi. org/10.21037/jtd-20-2122

Data Sharing Statement: Available at http://dx.doi. org/10.21037/jtd-20-2122

Peer Review File: Available at http://dx.doi.org/10.21037/jtd20-2122

Conflicts of Interest: All authors have completed the ICMJE uniform disclosure form (available at http://dx.doi. org/10.21037/jtd-20-2122). The authors have no conflicts of interest to declare.

Ethical Statement: The authors are accountable for all aspects of the work in ensuring that questions related to the accuracy or integrity of any part of the work are appropriately investigated and resolved. The study was 
conducted in accordance with the Declaration of Helsinki (as revised in 2013) and the Harmonized Tripartite Guideline for Good Clinical Practice from the International Conference on Harmonization. The study was reviewed and approved by the local ethics committee of the Hannover Medical School (Chair: Prof. S. Engeli, No. 8818_BOK_2019) and individual consent for this retrospective analysis was waived.

Open Access Statement: This is an Open Access article distributed in accordance with the Creative Commons Attribution-NonCommercial-NoDerivs 4.0 International License (CC BY-NC-ND 4.0), which permits the noncommercial replication and distribution of the article with the strict proviso that no changes or edits are made and the original work is properly cited (including links to both the formal publication through the relevant DOI and the license). See: https://creativecommons.org/licenses/by-nc-nd/4.0/.

\section{References}

1. Hoksch B, Birken-Bertsch H, Müller JM. Thoracoscopy before Jacobaeus. Ann Thorac Surg 2002;74:1288-90.

2. Bjork VO, Carlens $\mathrm{E}$. The prevention of spread during pulmonary resection by the use of a double-lumen catheter. J Thorac Surg 1950;20:151-7.

3. McKenna RJ Jr, Houck W, Fuller CB. Video-assisted thoracic surgery lobectomy: experience with 1,100 cases. Ann Thorac Surg 2006;81:421-5; discussion 425-6.

4. Pompeo E, Mineo D, Rogliani P, et al. Feasibility and results of awake thoracoscopic resection of solitary pulmonary nodules. Ann Thorac Surg 2004;78:1761-8.

5. Al-Abdullatief M, Wahood A, Al-Shirawi N, et al. Awake anaesthesia for major thoracic surgical procedures: an observational study. Eur J Cardiothorac Surg 2007;32:346-50.

6. Gonzalez-Rivas D, Paradela M, Fernandez R, et al. Uniportal video-assisted thoracoscopic lobectomy: two years of experience. Ann Thorac Surg 2013;95:426-32.

7. Gonzalez-Rivas D, Fernandez R, de la Torre M, et al. Single-port thoracoscopic lobectomy in a nonintubated patient: the least invasive procedure for major lung resection? Interact Cardiovasc Thorac Surg 2014;19:552-5.

8. Gonzalez-Rivas D, Aymerich H, Bonome C, et al. From Open Operations to Nonintubated Uniportal VideoAssisted Thoracoscopic Lobectomy: Minimizing the Trauma to the Patient. Ann Thorac Surg 2015;100:2003-5.

9. Liu J, Cui F, Li S, Chen H, et al. Nonintubated video- assisted thoracoscopic surgery under epidural anesthesia compared with conventional anesthetic option: a randomized control study. Surg Innov 2015;22:123-30.

10. Bertolaccini L, Zaccagna G, Divisi D, et al. Awake nonintubated thoracic surgery: an attempt of systematic review and meta-analysis. Video-assist Thorac Surg 2017;2:59.

11. Yu MG, Jing R, Mo YJ, et al. Non-intubated anesthesia in patients undergoing video-assisted thoracoscopic surgery: A systematic review and meta-analysis. PLoS One 2019;14:e0224737.

12. Wu CY, Chen JS, Lin YS, et al. Feasibility and safety of nonintubated thoracoscopic lobectomy for geriatric lung cancer patients. Ann Thorac Surg 2013;95:405-11.

13. Chen KC, Cheng YJ, Hung MH, et al. Nonintubated thoracoscopic lung resection: a 3-year experience with 285 cases in a single institution. J Thorac Dis 2012;4:347-51.

14. AlGhamdi ZM, Lynhiavu L, Moon YK, et al. Comparison of non-intubated versus intubated video-assisted thoracoscopic lobectomy for lung cancer. J Thorac Dis. 2018;10:4236-43.

15. Pompeo E, Sorge R, Akopov A, et al.; ESTS Nonintubated Thoracic Surgery Working Group. Nonintubated thoracic surgery-A survey from the European Society of Thoracic Surgeons. Ann Transl Med 2015;3:37.

16. Kaufmann KB, Loop T, Heinrich S, et al. Risk factors for post-operative pulmonary complications in lung cancer patients after video-assisted thoracoscopic lung resection: Results of the German Thorax Registry. Acta Anaesthesiol Scand 2019;63:1009-18.

17. Furák J, Szabó Z, Horváth T, et al. Non-intubated, uniportal, video-assisted thoracic surgery [VATS] lobectomy, as a new procedure in our department. Magy Seb 2017;70:113-7.

18. Forero M, Adhikary SD, Lopez H, et al. The Erector Spinae Plane Block: A Novel Analgesic Technique in Thoracic Neuropathic Pain. Reg Anesth Pain Med 2016;41:621-7.

19. Canet J, Gallart L, Gomar C, et al.; ARISCAT Group. Prediction of postoperative pulmonary complications in a population-based surgical cohort. Anesthesiology 2010;113:1338-50.

20. Neto AS, da Costa LGV, Hemmes SNT, et al.; LAS VEGAS Group. The LAS VEGAS risk score for prediction of postoperative pulmonary complications: An observational study. Eur J Anaesthesiol 2018;35:691-701.

21. Brunelli A, Varela G, Salati M, et al. Recalibration of the revised cardiac risk index in lung resection candidates. Ann Thorac Surg 2010;90:199-203. 
22. Zardo P, Zinne N, Logemann F, et al. Non-intubated uniportal video-assisted thoracoscopic surgery-lobectomy in Germany-the first 2 cases. J Vis Surg 2018;4:178.

23. Kregenow DA, Swenson ER. The lung and carbon dioxide: implications for permissive and therapeutic hypercapnia. Eur Respir J 2002;20:6-11.

24. Wu Y, Yang R, Xu J, et al. Effects of Intraoperative Fluid Management on Postoperative Outcomes After Lobectomy. Ann Thorac Surg 2019;107:1663-9.

25. Jeon CS, Yoon DW, Moon SM, Shin S, Cho JH et al. Non-intubated video-assisted thoracoscopic lung biopsy for interstitial lung disease: a single-center experience. J Thorac Dis 2018;10:3262-8.

26. Gonzalez-Rivas D, Bonome C, Fieira E, et al. Nonintubated video-assisted thoracoscopic lung resections: the future of thoracic surgery? Eur J Cardiothorac Surg 2016;49:721-31.

27. Hung MH, Hsu HH, Cheng YJ, et al. Nonintubated thoracoscopic surgery: state of the art and future directions. J Thorac Dis. 2014;6:2-9.

28. Chen KC, Cheng YJ, Hung MH, et al. Nonintubated thoracoscopic surgery using regional anesthesia and vagal block and targeted sedation. J Thorac Dis 2014;6:31-6.

29. Wang ML, Galvez C, Chen JS, et al. Non-intubated single-incision video-assisted thoracic surgery: a two-center cohort of 188 patients. J Thorac Dis. 2017;9:2587-98.

30. Zollinger A. Anästhesie in der Thoraxchirurgie [Anesthesia in thoracic surgery]. Anaesthesist 1999;48:193-204.

31. Fischer GW, Cohen E. An update on anesthesia for thoracoscopic surgery. Curr Opin Anaesthesiol 2010;23:7-11.

32. Zardo P, Busk H, Kutschka I. Chest tube management: state of the art. Curr Opin Anaesthesiol 2015;28:45-9.

33. Batchelor TJP, Rasburn NJ, Abdelnour-Berchtold E, et al. Guidelines for enhanced recovery after lung surgery: recommendations of the Enhanced Recovery After Surgery (ERAS®) Society and the European Society of Thoracic Surgeons (ESTS). Eur J Cardiothorac Surg 2019;55:91-115.

34. Pompili C, Falcoz PE, Salati M, et al. A risk score to predict the incidence of prolonged air leak after videoassisted thoracoscopic lobectomy: An analysis from the European Society of Thoracic Surgeons database. J Thorac Cardiovasc Surg 2017;153:957-65.

35. Júnior Ade P, Erdmann TR, Santos TV, et al. Comparison between continuous thoracic epidural and paravertebral blocks for postoperative analgesia in patients undergoing thoracotomy: Systematic review. Braz J Anesthesiol 2013;63:433-42.
36. Liu HY, Chiang XH, Hung MH, et al. Nonintubated uniportal thoracoscopic segmentectomy for lung cancer. J Formos Med Assoc. 2020;119:1396-1404.

37. Emelife PI, Eng MR, Menard BL, et al. Adjunct medications for peripheral and neuraxial anesthesia. Best Pract Res Clin Anaesthesiol 2018;32:83-99.

38. Weerink MAS, Struys MMRF, Hannivoort LN, et al. Clinical Pharmacokinetics and Pharmacodynamics of Dexmedetomidine. Clin Pharmacokinet 2017;56:893-913.

39. Lodenius $\AA$, Maddison KJ, Lawther BK, et al. Upper Airway Collapsibility during Dexmedetomidine and Propofol Sedation in Healthy Volunteers: A Nonblinded Randomized Crossover Study. Anesthesiology 2019;131:962-73.

40. Jakob SM, Ruokonen E, Grounds RM, et al.; Dexmedetomidine for Long-Term Sedation Investigators. Dexmedetomidine vs. midazolam or propofol for sedation during prolonged mechanical ventilation: two randomized controlled trials. JAMA 2012;307:1151-60.

41. Iwata Y, Hamai Y, Koyama T. Anesthetic management of nonintubated video-assisted thoracoscopic surgery using epidural anesthesia and dexmedetomidine in three patients with severe respiratory dysfunction. J Anesth 2016;30:324-7.

42. Hung MH, Hsu HH, Chan KC, et al. Non-intubated thoracoscopic surgery using internal intercostal nerve block, vagal block and targeted sedation. Eur J Cardiothorac Surg 2014;46:620-5.

43. Lai HC, Huang TW, Tseng WC, et al. Sevoflurane is an effective adjuvant to propofol-based total intravenous anaesthesia for attenuating cough reflex in nonintubated video-assisted thoracoscopic surgery. Medicine (Baltimore) 2018;97:e12927.

44. Smetana GW, Lawrence VA, Cornell JE, et al. Preoperative pulmonary risk stratification for noncardiothoracic surgery: systematic review for the American College of Physicians. Ann Intern Med 2006;144:581-95.

45. Agostini P, Cieslik H, Rathinam S, et al. Postoperative pulmonary complications following thoracic surgery: are there any modifiable risk factors? Thorax 2010;65:815-8.

46. Brunelli A, Varela G, Van Schil P, et al. Multicentric analysis of performance after major lung resections by using the European Society Objective Score (ESOS). Eur J Cardiothorac Surg 2008;33:284-8.

47. Pompeo E, Rogliani P, Tacconi F, et al. Randomized comparison of awake nonresectional versus nonawake resectional lung volume reduction surgery. J Thorac Cardiovasc Surg 2012;143:47-54. 
48. Tacconi F, Pompeo E. Non-intubated video-assisted thoracic surgery: where does evidence stand? J Thorac Dis 2016;8:S364-75.

49. Deng HY, Zhu ZJ, Wang YC, et al. Non-intubated video-assisted thoracoscopic surgery under loco-regional anaesthesia for thoracic surgery: a meta-analysis. Interact Cardiovasc Thorac Surg 2016;23:31-40.

50. Della Rocca G, Coccia C. Acute lung injury in thoracic surgery. Curr Opin Anaesthesiol 2013;26:40-6.

51. Hausman MS Jr, Jewell ES, Engoren M. Regional versus

Cite this article as: Starke H, Zinne N, Leffler A, Zardo P, Karsten J. Developing a minimally-invasive anaesthesiological approach to non-intubated uniportal video-assisted thoracoscopic surgery in minor and major thoracic surgery. J Thorac Dis 2020;12(12):7202-7217. doi: 10.21037/jtd-20-2122 general anesthesia in surgical patients with chronic obstructive pulmonary disease: does avoiding general anesthesia reduce the risk of postoperative complications? Anesth Analg 2015;120:1405-12.

52. Kirmeier E, Eriksson LI, Lewald H, et al. POPULAR Contributors. Post-anaesthesia pulmonary complications after use of muscle relaxants (POPULAR): a multicentre, prospective observational study. Lancet Respir Med 2019;7:129-40. 